\title{
Global mapping of landscape fragmentation, human-animal interactions, and livelihood behaviors to prevent the next pandemic
}

\author{
Laura S. P. Bloomfield ${ }^{1,2}$
}

Accepted: 25 April 2020 / Published online: 13 May 2020

(c) Springer Nature B.V. 2020

Everyone wants to know when the next pandemic will occur. Scientists have long anticipated the emergence of the next infectious agent, but none could have foreseen the unprecedented biological, social, and economic devastation of COVID-19. Research on zoonoses has often focused on investigating viral diversity in potential animal hosts in areas considered "hotspots" as the approach to predicting, preventing, or quickly responding to a spillover event. While these impressive viral studies have helped us to better understand the propensity of wildlife to share infections with people (and people with animals), these scientific insights are unlikely to provide adequate information to forecast the emergence of the next infectious agent or guidance on how to best intervene. As a researcher who performed a viral study in Uganda, a place considered a potential zoonotic hotspot, I believe that investing in landscape-based behavioral research and site-specific interventions hold greater promise for mitigating spillover risk.

Studies on viral diversity frequently acknowledge the potential low yield of widespread viral monitoring because the majority of viruses that pass from wild animals into humans do not survive and infect human cells or cause systemic disease. As far as we know, even fewer of these animal viruses can be passed between people. Therefore, to locate the precursor infection in a human or animal host prior to a spillover event and intervene in a timely manner is incredibly unlikely. Viruses are constantly circulating,

This article is part of the TopicalCollection: Agriculture, Food \& Covid-19.

Laura S. P. Bloomfield

labloom@stanford.edu

1 Stanford University School of Medicine, Stanford University, Stanford, CA 94305, USA

2 Emmett Interdisciplinary Program in Environment and Resources, Stanford University, Stanford, CA 94305, USA evolving, and being passed between hosts. Thus, we are looking for a needle in a haystack. Instead, perhaps we should be looking at how the haystacks are arranged and how their composition influences the distribution and interaction of their needles.

Strong evidence points to human-animal interactions as the source of COVID-19 as well as past pandemics, including SARS-CoV, MERS-CoV, and Ebola, to name a few. Indeed, it is likely that the next infectious agent to adversely affect humanity will have animal origins. Our recent study in Uganda (Bloomfield et al. 2020) explicitly linked landscape fragmentation and forest resource collection to an increased likelihood of contact between people and nonhuman primates. Therefore, increased fragmentation of animal habitats interspersed with developed agricultural land puts vulnerable populations at ever-increasing risk of zoonotic exposure. These fragmented landscapes also lead to poor outcomes for wild nonhuman primates which are often viewed as pests in these contexts and are at increased risk of being maimed, hunted or even acquiring infections from people.

Insights into the complex dynamics influencing humananimal contact events in Uganda are applicable to other biodiverse areas with dense human populations and intensified pressure on natural landscapes. With a world population nearing an estimated 8 billion, we will likely see increasingly fragmented landscapes in which people and wild animals overlap. Creating a global map of the most common types, frequencies, intensities, and locations of human-animal interactions may allow more intentional selection of conservation corridors, creation of buffer zones between human and wild animal habitats, and support of local livelihoods. With local understanding of the environmental, economic, and social pressures leading to humananimal interactions, we have a real opportunity to prevent contact, subsequent viral spillover, and hopefully, the next pandemic. 


\section{Reference}

Bloomfield, Laura S.P., Tyler L. McIntosh, and Eric Lambin. 2020. Habitat fragmentation, livelihood behaviors, and contact between people and nonhuman primates in Africa. Landscape Ecology. https://doi.org/10.1007/s10980-020-00995-w.

Publisher's Note Springer Nature remains neutral with regard to jurisdictional claims in published maps and institutional affiliations.
Laura S.P. Bloomfield is a MD/PhD candidate in the School of Medicine and the Emmett Interdisciplinary Program in Environment and Resources at Stanford University where her research focuses on the influence of land-use change and social network structures on infectious disease transmission. Shehas a particular interest in biodiverse places undergoing deforestation and working with people who are most vulnerable to zoonotic spillover. Laura holds a MS in Earth Systems Science and a BA in Anthropological Science from Stanford University. 